\title{
Adaptação transcultural da Heartland Forgiveness Scale (HFS) em amostra de idosos brasileiros
}

\author{
Adaptación transcultural de la Heartland Forgiveness Scale (HFS) en muestra de \\ mayores brasileños
}

\section{The Heartland Forgiveness Scale (HFS) cross-cultural adaptation in a brazilian elderly sample}

Gabriela Veiga Alano Rodrigues Pontifícia Universidade Católica do Rio Grande do Sul (PUCRS), Porto Alegre - RS/Brasil ORCID: 0000-0001-6074-7905

E-mail: gabriela.veiga@edu.pucrs.br

Carolina Villanova Quiroga

Pontifícia Universidade Católica do Rio Grande do Sul (PUCRS), Porto Alegre - RS/Brasil ORCID: 0000-0001-8946-8897

E-mail: carolina.quiroga@acad.pucrs.br

Irani Iracema de Lima Argimon Pontifícia Universidade Católica do Rio Grande do Sul (PUCRS), Porto Alegre - RS/Brasil ORCID: 0000-0003-4984-0345

E-mail: argimoni@pucrs.br

\begin{abstract}
Resumo
A Heartland Forgiveness Scale (HFS) é um instrumento que mede a disposição pessoal para o perdão em relação aos outros, a si mesmo e a situações fora do controle de qualquer pessoa. O presente estudo teve como objetivo traduzir e adaptar transculturalmente a HFS para idosos brasileiros. Para tanto, o método de adaptação do instrumento foi divido em etapas: definições conceituais e revisão da literatura; tradução e síntese das traduções; retrotradução (backtraslation); análise de especialistas; estudo piloto e elaboração da versão final do instrumento; primeiras análises psicométricas da versão final. Nas etapas de tradução, retrotradução e análises de especialistas participaram quatro tradutores e duas psicólogas especialistas. Já o estudo piloto contou com a participação de 32 idosos provenientes da região Sul do Brasil, com idades entre 60 e 89 anos, em sua maioria com alto grau de escolaridade. Utilizou-se uma Ficha de dados sócio demográficos e a HFS. A partir desse estudo, ainda que em caráter inicial, pode-se concluir que a HFS está adaptada transculturalmente para a população idosa brasileira. Sendo assim, está apta a ser aplicada em uma amostra maior para que possa ser submetida a análises psicométricas de sua validação. A adaptação de instrumentos de outras culturas nessa temática se faz necessária para realidade brasileira devido a escassez de estudos na área do perdão, em especial com populações de idosos. Futuramente, pode também contribuir para a elaboração de técnicas de intervenção e criação de estratégias voltadas para prevenção e promoção da saúde do idoso.
\end{abstract}

Palavras-chaves: Idosos; Perdão; Adaptação transcultural.

\section{Resumen}

La Escala de Perdón de Heartland (HFS) es un instrumento que mide la disposición de una persona a perdonar en relación con los demás, con uno mismo y situaciones que están fuera del control de cualquier persona. El presente estudio tuvo como objetivo traducir y adaptar transculturalmente la HFS para la población de mayores brasileños. Para tanto, el método de adaptación se dividió en etapas: definiciones conceptuales y revisión de literatura; traducción y síntesis de traducciones; retrotraducción; análisis de expertos; estudio piloto y elaboración de la versión final del instrumento; primeros análisis psicométricos de la versión final. En las etapas de traducción, retrotraducción y análisis experto 
participaron cuatro traductores y dos psicólogos expertos. El estudio piloto contó con la participación de 32 mayores de la región sur de Brasil, con edades entre 60 y 89 años, la mayoría con nivel educativo alto. Se utilizó una ficha sociodemográfica y la HFS. De este estudio, aunque en un carácter inicial, se puede concluir que el HFS está adaptada transculturalmente para la población anciana brasileña. Por tanto, se puede aplicar en una muestra mayor para que pueda ser sometido a análisis psicométricos para su validación. La adaptación de instrumentos de otras culturas en este tema es necesaria para la realidad brasileña debido a la escasez de estudios en el área del perdón, especialmente con esta poblacion. En el futuro, también puede contribuir al desarrollo de técnicas de intervención y creación de estrategias dirigidas a prevenir y promover la salud de los mayores.

Palabras clave: Anciano; Perdón; Adaptación transcultural.

\begin{abstract}
The Heartland Forgiveness Scale (HFS) is an instrument that measures personal willingness to forgive in relation to others, yourself and situations that are beyond anyone's control. The present study aimed to translate and cross-culturally adapt HFS for the elderly Brazilian population. Therefore, the method was divided into
\end{abstract}

stages: conceptual definitions and literature review; translation and synthesis of translations; backtranslation; expert analysis; pilot study and preparation of the final version of the instrument; first psychometric analyzes of the final version of the instrument. In the translation, backtranslation and expert analysis stages, four translators and two specialist psychologists participated. The pilot study had the participation of 32 elderly people from the southern region of Brazil, aged between 60 and 89 years, most of them with a high level of education. The instruments used were: socio-demographic data sheet and the HFS. From this study, although in an initial character, it can be concluded that HFS is translated into Brazilian Portuguese and adapted cross-culturally for the elderly Brazilian population. Therefore, it is able to be applied to a larger sample so it's possible to carry on psychometric analysis of its validation. Other cultures instrument adaptation on this theme are necessary for the Brazilian reality due to the scarcity of instruments about forgiveness, especially in the elderly population. In the future, it can also contribute to the development of intervention techniques and the creation of strategies aimed at preventing and promoting the health of the elderly.

Keywords: Elderly; Forgiveness; Transcultural adaptation.

\section{Introdução}

Cada vez mais se observa um crescente investimento no desenvolvimento de pesquisas que contemplem estudos sobre o envelhecimento, bem como elaboração de estratégias que possibilitem e facilitem um envelhecimento bem sucedido. Este movimento da comunidade científica se dá pela observação do acentuado envelhecimento populacional a partir de importantes mudanças demográficas a nível mundial. Atualmente os idosos representam $12 \%$ da população geral, e há projeções deste número duplicar até 2050 (Veras, 2009; Suzman, Beard, Boerma, \& Chatterji, 2015).

\section{Idosos representam um grupo} populacional mais vulnerável a declínio nos níveis de felicidade e bem-estar subjetivo. Isto se dá pelas diversas perdas normativas em diferentes domínios relacionadas ao processo de envelhecimento, tais como perda auditiva, dificuldades físicas, isolamento social decorrente da aposentadoria e dificuldade de locomoção, por exemplo. Esta tendência pode levar a uma série de condições de adoecimento mental, tais como ansiedade e depressão (Godoy-Izquierdo, Martínez, \& Godoy, 2009). Assim, faz-se importante o desenvolvimento de intervenções que permitam a manutenção de bem-estar físico e mental desta população. Nesse contexto, a organização de um sistema eficiente de atenção a saúde do idoso, que vise qualidade de vida de maneira global, é um dos principais desafios atuais.

\section{A Organização Mundial da Saúde} (OMS, 2002), em contribuição a segunda Assembléia Mundial das Nações Unidas sobre Envelhecimento, definiu o envelhecimento saudável como um processo no qual o indivíduo possa desenvolver e manter sua capacidade funcional, permitindo assim um bem-estar na idade avançada. Entende-se por capacidade funcional a associação entre capacidades físicas, mentais e psicossociais, garantindo um nível satisfatório de autonomia 
e independência, atentando para o bem-estar subjetivo do idoso.

Entendendo o bem estar subjetivo como um conjunto multifatorial, faz-se importante aprimorar características que possam estimular o idoso na manutenção da sua qualidade de vida, independentemente das limitações comumente decorrentes dessa fase vital. A Psicologia Positiva surge como uma vertente teórica da psicologia que trabalha o desenvolvimento e fortalecimento de diferentes forças e virtudes, estas podendo impulsionar um envelhecimento psicossocial mais saudável (Rodrigues, Giacomoni, \& Argimon, 2019). Dentre os objetos de estudo da Psicologia Positiva se encontra o Perdão, constructo este correlacionado positivamente com longevidade (Toussaint, Owen, \& Cheadle, 2012).

Ao longo da história, diferentes autores partem de perspectivas distintas sobre a definição do processo de perdão. A Psicologia Positiva, a partir de uma perspectiva multidisciplinar, considera o Perdão como um processo que envolve ferramentas cognitivas, emocionais, motivacionais e sociais. Para Seligman (2002), o perdão é uma das atitudes que permitem mudar o foco das experiências negativas e gerar emoções positivas, sendo capaz assim de aumentar a satisfação do indivíduo e o sentimento em relação a ação do outro.

Quanto ao tipo de respostas a situações negativas Thompson et al. (2005) explicam que não é necessário que o indivíduo desenvolva respostas positivas, como compaixão e empatia frente a transgressão, ou seja, a situação ou pessoa responsável pelos sentimentos ruminativos. Se ele desenvolver respostas neutras, estas já são consideradas suficientes para se dar o processo de perdão.

De maneira geral as medidas que avaliam a propensão para perdoar são correlacionadas positivamente com medidas de saúde mental e bem estar. Ademais, pessoas que perdoam mais apresentam escores mais baixos em medidas de níveis de ansiedade, sintomatologia depressiva e hostilidade, assim como menores escores de ruminação e narcisismo, e maior empatia (McCullough \& Witvliet, 2002).

Já foram desenvolvidos estudos que buscaram entender a influência da idade na propensão para perdoar, concluindo que as pessoas aparentam estarem mais abertas a perdoar conforme vão envelhecendo. Ademais, o Perdão tem se mostrado um importante recurso para lidar com déficits inerentes ao processo de envelhecimento (Allemand, 2008; Ramírez, Ortega, Chamorro, \& Colmenero, 2014).

Buscando avaliar a tendência geral de um indivíduo para perdoar, a escala Heartland Forgiveness Scale - HFS foi desenvolvida por Thompson et al. em 2005. Trata-se de um questionário de auto relato composto por 18 itens divididos em três subescalas: auto-perdão (itens 1-6), perdão do outro (itens 7-12) e perdão de situações além do controle de qualquer pessoa (itens 13-18). A versão original da HFS demonstrou validade de convergência, consistência interna satisfatória e forte confiabilidade teste-reteste.

A HFS já foi traduzida para mais de 20 idiomas, sendo adaptada e validada para diferentes populações. Pesquisadores de países como Espanha (Gallo-Giunzioni, Prieto-Ursúa, Fernández-Belinchón, \& Luque-Reca, 2021), Portugal (Ikedo, Castro, Fraguas, Rego, \& Nunes, 2020), India (Dahiya \& Rangnekar, 2020), entre diversos outros, já adaptaram a escala para suas populações. O processo de tradução e adaptação de instrumentos é de extrema relevância científica, pois permite a comparação de resultados de um mesmo instrumento em amostras distintas. Devem ser considerados aspectos culturais, idiomáticos, linguísticos e contextuais ao adaptar um instrumento, para que atenda a demanda da população em questão (Borsa, Damásio, \& Bandeira, 2012).

Considerando o aporte teórico, este trabalho objetiva apresentar o processo de adaptação transcultural da Heartland Forgiveness Scale (HFS) para idosos 
brasileiros. Ademais, busca-se apresentar dados estatísticos preliminares quanto à consistência interna da adaptação brasileira.

\section{Método Participantes}

$\mathrm{Na}$ etapa de tradução do instrumento, participaram dois tradutores bilíngues português-inglês, conforme orientações de Beaton, Bombardier, Guillemin e Ferraz (2002). Estes tinham como língua mãe o português brasileiro, mas possuíam o inglês como segundo idioma. $\mathrm{Na}$ etapa da Retrotradução participaram mais dois tradutores bilíngues, porém estes tinham o inglês como língua mãe e o português brasileiro como segundo idioma. Já na etapa de avaliação por experts, participaram duas psicólogas especialistas. Os critérios para serem consideradas experts foram: trabalhar com Psicologia Positiva e possuir publicações na área.

Já no estudo piloto participaram 32 indivíduos a fim de verificar a adequabilidade das instruções da HFS, além de estimar o tempo de administração da tarefa (Cooper et al., 2012; Pasquali, 2016). Como critério de exclusão foi considerada a impossibilidade de responder o instrumento devido a percepção de déficit cognitivo do participante por avaliação qualitativa do pesquisador aplicador. Nesta etapa não houveram participantes que se negaram a colaborar com o estudo ou que desistiram da participação durante a fase de coleta, bem como perda amostral por instrumento respondido de forma errônea.

\section{Procedimentos}

O processo de adaptação se deu em um total de oito etapas (figura 1). Inicialmente foi realizado o contato via e-mail com a autora da HFS, informando o interesse em adaptar esse instrumento para a população idosa brasileira. Esta concedeu autorização para a tradução, adaptação transcultural e uso em contexto brasileiro. A adaptação transcultural do HFS seguiu a Resolução 196/96 do Conselho Nacional de Saúde para pesquisas com seres humanos, e o projeto foi aprovado pelo Comitê de Ética em Pesquisa da PUCRS, sob CAE 80116817.0.0000.5336.

Os participantes do estudo piloto assinaram o Termo de Consentimento Livre e Esclarecido (TCLE), sendo informados do objetivo da atividade e das questões éticas e de sigilo em relação aos seus dados. Inicialmente, foi estabelecido o rapport e a explicação sobre os procedimentos da pesquisa com a leitura e assinatura do TCLE. Em seguida, os participantes responderam aos instrumentos da bateria de avaliação. Os participantes da pesquisa foram avaliados individualmente em um encontro de aproximadamente 40 minutos, as avaliações foram conduzidas por uma equipe de psicólogos e estudantes da graduação em Psicologia capacitados previamente na administração, registro e correção dos instrumentos.

Os instrumentos aplicados no estudo piloto, nesta ordem, foram:

Ficha de dados socio-demográficos e de saúde: a ficha de dados sócio-demográficos incluiu variáveis demográficas e de saúde, tais como: idade, sexo, estado civil, escolaridade, espiritualidade, renda, situação de moradia, ocupação atual, atividades de lazer, estado subjetivo de saúde física e mental, uso de medicação, uso de tabaco e bebida (quantidade e frequência), atividade física realizada e participação em grupos de idosos. Esta ficha foi desenvolvida pelos pesquisadores responsáveis pelo estudo especificamente para adaptação deste instrumento.

Heartland Forgiveness Scale (HFS): trata de um questionário de 18 itens que mede a disposição para Perdoar de uma pessoa (isto é, a tendência geral de perdoar), em vez do perdão de um evento ou pessoa em particular. A HFS consiste no escore Total HFS e três subescalas de seis itens cada, sendo estas Auto Perdão, Perdão dos Outros e Perdão das Situações. Elaborada por Thompson et al. (2003). Trata-se de uma escala Likert de 7 pontos, variando entre "quase sempre falso", onde o escore é 
zero, e "quase sempre verdadeiro", sendo o escore máximo (Thompson et al., 2005).

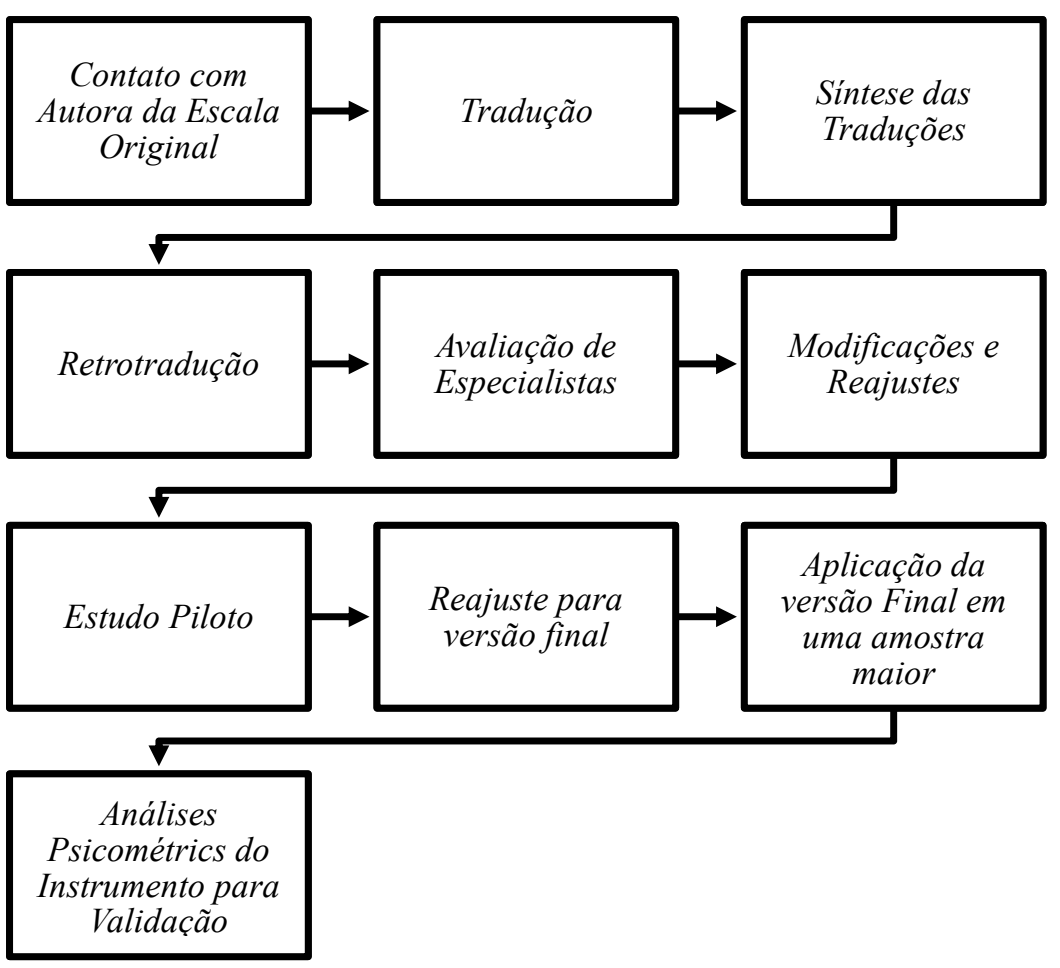

Figura 1. Fluxograma - Etapas da Pesquisa

Fonte: Fluxograma elaborado pelas autoras, 2017.

\section{Análise de dados}

Após conclusão da versão final do instrumento foram realizadas análises estatísticas com base no estudo piloto. Para análise dos dados obtidos foi utilizado o pacote estatístico SPSS versão 22 para Windows. Em um primeiro momento foi verificado especificamente o Alpha de Cronbach da escala, a fim de validar o processo de adaptação através de avaliação de consistência interna sendo considerados valores apropriados iguais ou acima de 0.70 tanto para escore total quanto para cada uma das subescalas. Os dados foram analisados através de estatísticas descritivas (média, desvio-padrão, percentuais e percentis) e inferencial. Na análise de conteúdo realizada pelos profissionais de Psicologia, o índice de concordância foi avaliado através do método de Fagundes (1999). No estudo piloto, os resultados foram analisados também qualitativamente (dificuldades apresentadas pelos participantes, adequabilidade das instruções e dos estímulos, por exemplo).

\section{Resultados Tradução}

Nessa etapa, dois profissionais bilíngues (tradutores) traduziram, de forma independente, a HFS para o idioma português brasileiro. Optou-se por dois tradutores com intuito de diminuir o risco de possíveis vieses de tradução diferenciada devido a linguística ou por motivos culturais. A participação deles na pesquisa foi voluntária e não remunerada (Borsa et al., 2012).

\section{Síntese das versões traduzidas}

Nessa etapa foram avaliadas as versões traduzidas de maneira a manter o significado igual ao da versão original em cada item. Para tanto, essa análise foi feita com os membros responsáveis pelo estudo, em parceria com os tradutores, elaborando assim uma síntese das traduções (Borsa et al., 2012). 
A síntese das traduções foi realizada levando em consideração o índice de concordância entre as versões traduzidas comparadas pela pesquisadora. Após consenso entre os tradutores e pesquisadores em relação aos itens divergentes, criou-se a versão brasileira inicial da HFS.

\section{Retrotradução ou Tradução Reversa}

Nesta etapa, tradutores que tem como língua materna o idioma da versão original, ou seja, o inglês americano, receberam a versão inicial da HFS (síntese das traduções) para que pudessem realizar o processo de retrotradução. Ou seja, as versões voltaram a ser traduzidas para o inglês "às cegas", uma vez que os tradutores em questão não tinham qualquer conhecimento sobre a versão original. Em seguida, realizou-se a síntese das retrotraduções pelos pesquisadoros, a fim de comparar com o instrumento original, verificando se havia incongruências (CasseppBorges, Balbinotti, M., \& Teodoro, 2010). Em caso de incongruência, entenderia-se que há necessidade de reiniciar o processo de tradução. Entretanto, verificou-se que a versão originária da retrotradução se mostrava semelhante a versão do instrumento original, não sendo necessário assim reiniciar a tradução.

\section{Avaliação por experts}

A versão final da HFS foi encaminhada para uma comissão de especialistas para a análise da clareza das instruções, assim como para realizar a análise de conteúdo do instrumento. Nessa etapa a comissão de especialistas avaliou o instrumento em quatro tópicos: semântico, idiomático, experimental e conceitual. Para tanto, foram convidadas duas profissionais especialistas da área de psicologia com experiência em Psicologia Positiva. Estas também puderam dar sugestões e indicar termos mais adequados que demonstrassem claramente o significado que se deseja, para que pudesse ser realizado o estudo piloto com a versão final (Hungerbühler \& Wang, 2016).

A participação das experts foi voluntária e sem remuneração. A partir da análise dos especialistas foram feitas sugestões na modificação de termos mais adequados à realidade da população em questão.

Foram avaliadas questões como: se as palavras eram equivalentes às da versão original; se a tradução teve alguma limitação gramatical; se foram usadas expressões voltadas para o contexto cultural da população idosa; questões de adequabilidade do instrumento para o público-alvo; se o instrumento estava compreensível e adequado para a população idosa de variada escolaridade; o tamanho da letra; e por fim, foi verificado se os conceitos dos termos usados possuíam concordância com o que se propõe a versão original (Hungerbühler \& Wang, 2016). As sugestões de modificações realizadas pelos profissionais foram discutidas entre as autoras.

Foi solicitado que os especialistas avaliassem a escala em diversos aspectos, desde o rapport apresentado nas instruções do instrumento e a clareza das instruções, até os aspectos internos da HFS, na análise semântica, idiomática e experimental. Em relação à equivalência semântica, os juízes avaliaram termos de significados múltiplos e quais poderiam se adequar melhor com o instrumento para o público-alvo. Nessa etapa, os juízes averiguaram se as traduções tinham consistência. Já na avaliação idiomática, eles observaram se a versão das traduções abarcava o significado de acordo com o contexto brasileiro, e não uma tradução simples e/ou literal.

A etapa seguinte se deu pela análise conceitual das palavras, em que foi avaliado se $o$ instrumento estava mensurando o aspecto do construto ao qual se propunha. Sendo assim, foi analisado se todos os itens da HFS estavam avaliando os fatores relacionados ao Perdão, observando assim se os itens do instrumento possuíam alguma questão que não se encaixava no objetivo proposto. Nesta etapa, os juízes avaliaram a consistência interna da HFS para julgar se havia intercorrelação entre seus itens e os três aspectos distintos do Perdão os quais o instrumento se propõe a avaliar. Por fim, foi analisado o grau de concordância das respostas 
entre os juízes, e após nova avaliação dos pesquisadores, ajustes foram realizados para dar seguimento na análise pelo público-alvo.

Algumas alterações foram realizadas após a análise dos juízes. Estas se deram especialmente em relação à adequação de termos e palavras para que se pudesse alcançar o objetivo proposto de mensurar o construto no público alvo (Tabela 1).

\section{Tabela 1}

Exemplos das modificações

\begin{tabular}{|c|c|c|}
\hline & Primeira Versão & Após revisão \\
\hline Item 1 & $\begin{array}{l}\text { "Ainda que incialmente eu me sinta mal } \\
\text { quando cometo um erro, com o passar o } \\
\text { tempo eu consigo relaxar." }\end{array}$ & $\begin{array}{l}\text { "Ainda que incialmente eu me sinta mal } \\
\text { quando cometo um erro, com o passar o } \\
\text { tempo eu consigo me sentir melhor." }\end{array}$ \\
\hline Item 11 & $\begin{array}{l}\text { "Se alguém me maltrata, eu continuo a } \\
\text { pensar mal dessa pessoa" }\end{array}$ & $\begin{array}{l}\text { "Se alguém me maltrata, com o passar do } \\
\text { tempo eu continuo a pensar mal dessa } \\
\text { pessoa" }\end{array}$ \\
\hline Item 16 & $\begin{array}{l}\text { "Eu eventualmente faço as pazes com } \\
\text { circunstâncias ruins na minha vida" }\end{array}$ & $\begin{array}{l}\text { "Eu acabo aceitando as circunstâncias ruins } \\
\text { que ocorreram na minha vida" }\end{array}$ \\
\hline Item 18 & $\begin{array}{l}\text { "Eventualmente eu paro de ter } \\
\text { pensamentos } \quad \text { negativos } \\
\text { circunstâncias ruins que estão além do } \\
\text { controle de qualquer pessoa." }\end{array}$ & $\begin{array}{l}\text { "Com o passar do tempo eu paro de ter } \\
\text { pensamentos negativos sobre circunstâncias } \\
\text { ruins que estão além do controle de qualquer } \\
\text { pessoa." }\end{array}$ \\
\hline
\end{tabular}

Nota. Tabela elaborada pelas autoras, 2019.

\section{Análise pelo Público Alvo}

Após a síntese das traduções, a HFS foi aplicada em um grupo, de cinco idosos, os quais responderam um questionário de seis itens sobre a clareza e dificuldades do instrumento. As quatro primeiras perguntas eram respondidas em escala tipo Likert de três respostas ("Sim", "Um pouco" e "Não") e as duas finais eram respondidas descritivamente. Essa etapa teve também como intuito mensurar a média de tempo para o manejo do instrumento e identificar possíveis adequações visuais necessárias (formato da escala likert e tamanho de fonte da letra, por exemplo).

\section{Estudo piloto}

O estudo piloto se constituiu em 32 idosos com idade igual ou superior a 60 anos, recrutados por conveniência, que responderam, de maneira individual, a primeira versão da HFS. A coleta se deu de forma presencial nas salas do Programa de Pós Graduação em Psicologia da PUCRS, sendo o instrumento aplicado por pesquisadores e estudantes de psicologia previamente treinados. Inicialmente era realizado rapport com explicação sobre os procedimentos da pesquisa e com a leitura e assinatura do Termo de Consentimento Livre e Esclarecido (TCLE). Durante a avaliação, aqueles participantes que demonstrarem dificuldades para responder aos questionários por problemas de compreensão ou por baixa 
escolaridade, receberam auxílio da equipe de pesquisa.

Os participantes responderam cada questão do instrumento e, quando necessário, informaram sobre suas dificuldades de compreensão, de adequação e clareza dos itens ou instrução (Hungerbühler \& Wang, 2016). Esta etapa serviu para verificar a compreensibilidade das instruções, além de certificar o tempo de administração previamente calculado.

Características sociodemográficas da amostra do Estudo Piloto

Os idosos que participaram do grupo piloto eram provenientes de diferentes regiões do estado do Rio Grande do Sul. A amostra se caracterizou por 32 idosos, 30 participantes do sexo feminino e apenas 2 do sexo masculino. As idades variaram entre 60 e 89 anos, em uma média de idade de 74 anos. Em relação à escolaridade, $28 \%$ da amostra possui ensino superior completo e $31 \%$ possui pós- graduação. Quanto ao estado civil, 53\% dos idosos entrevistados eram viúvos, enquanto $25 \%$ eram divorciados. Quando questionado a respeito da religião de eleição, $62 \%$ da amostra se declarou católica, $18 \%$ espírita e o restante dividiu-se em umbandista, ateu, judeu ou não considerava pertencer a nenhuma religião. Ressalta-se que $68 \%$ da amostra consideram a Espiritualidade como tendo muita importância na vida.

Para as análises estatísticas da HFS foi mensurada a consistência interna (ou seja, a concordância) entre os itens em termos de correlação entre eles (Streiner, Norman, \& Cairney, 2014). Segundo a literatura o valor deve ser igual ou acima de 0,70 (Cronbach, 1951). A escala HFS apresentou um valor adequado ( $\alpha=0,818)$, levando ainda em consideração o $\mathrm{N}$ do estudo piloto, indicando boa consistência interna da escala. Quando avaliadas separadamente as subescalas da HFS apresentaram os seguintes valores: Subescala 1 - $\alpha=0,547$, Subescala $2-\alpha=0,726$ e Subescala $3-\alpha=0,492$ (Tabela 2).

\section{Tabela 2}

Alpha de Cronbach da HFS por item

\begin{tabular}{l|l|l} 
& $\begin{array}{l}\text { Alpha de Cronbach se o item for } \\
\text { deletado }\end{array}$ & $\begin{array}{l}\text { Alpha de Cronbach se o item for deletado } \\
\text { (subescalas analisadas separadamente) }\end{array}$ \\
\hline HFS_1 &, 821 &, 603 \\
\hline HFS_2 &, 797 &, 380 \\
\hline HFS_3 &, 812 &, 538 \\
\hline HFS_4 &, 819 &, 576 \\
\hline HFS_5 &, 818 &, 375 \\
\hline HFS_6 &, 804 &, 624 \\
\hline HFS_7 &, 791 &, 710 \\
\hline HFS_8 &, 809 &, 617 \\
\hline HFS_9 &, 799 &, 724 \\
\hline HFS_10 &, 809 &, 664 \\
\hline HFS_11 &, 798 &, 746 \\
\hline HFS_12 &, 814 &, 228 \\
\hline HFS_13 &, 789 & \\
\hline
\end{tabular}




\begin{tabular}{l|l|l}
\hline HFS_14 &, 817 &, 520 \\
\hline HFS_15 &, 804 &, 350 \\
\hline HFS_16 &, 831 &, 634 \\
\hline HFS_17 &, 808 &, 310 \\
\hline HFS_18 &, 813 &, 456 \\
\hline
\end{tabular}

Nota. Tabela elaborada pelas autoras, 2019.

${ }^{a}$ A HFS - agora nominada no Brasil como Escala Heartland do Perdão - foi enviada aos autores do instrumento original, devidamente autorizada e se encontra publicada no site oficial www.heartlandforgiveness.com, disponível para uso.

\section{Discussão}

Este estudo teve como objetivo apresentar o processo de tradução e adaptação transcultural da HFS para o português brasileiro, utilizando uma amostra de idosos do sul do país. Durante o estudo piloto não foram verbalizadas dúvidas dos participantes em relação aos itens relatados, o que demonstra um processo de tradução adequado. Ademais, qualitativamente, não foram observadas demandas para alterações no instrumento. Sendo assim optou-se por manter a versão do estudo piloto como versão final do instrumento, para que posteriormente possa ser aplicado em uma amostra maior, visando análises psicométricas de validação mais robustas.

Quanto à análise dos juízes não especialistas, após reajustes, os participantes declararam que a HFS estava clara e adequada em seus itens. É importante ressaltar que por ser uma população com algumas limitações devido a declínios naturais do processo de envelhecimento, exige maiores adaptações quando comparada ao público-alvo em que a escala original foi aplicada (universitários). A escala que originalmente foi pensada em ser auto respondida, podendo ser aplicada de forma grupal, demonstrou em sua versão adaptada para idosos a necessidade de auxílio durante a aplicação. Sendo assim se sugere que seja aplicada de forma individual junto a um aplicador treinado.

Considerando aspectos psicométricos de adaptação, a escala original apresenta consistência interna de $\alpha=0.87$ (Thompson et al., 2005). Já a escala HFS versão traduzida para o português brasileiro apresentou valor de $\alpha=0.818$, estando em consonância com o recomendável pela literatura científica. Ao comparar as duas escalas, podemos observar certa similaridade dos números, o que é considerado um bom resultado dentro da psicometria (Artes \& Barroso, 2016).

Instrumentos validados para amostras de idosos trazem a necessidade de, por vezes, revisar a quantidade de itens de opções de respostas, visto dificuldades geradas por diferentes características desta população. Percebeu-se neste estudo algumas dificuldades da amostra em compreender a escala likert de sete itens. Sugere-se revisão do número de itens da escala likert e uma possível futura redução nas opções de respostas, após estudos com amostras robustas, clínicas e não clínicas. Porém, em geral, na avaliação pelo públicoalvo, a escala obteve alta concordância dos juízes não especialistas sobre sua clareza e adequação, esta mensurada de forma qualitativa.

Cabe destacar limitações deste estudo. Por se tratar de uma amostra pequena se impossibilitou realizar analises psicométricas mais robustas para iniciar o processo de validação. As particularidades da populaçãoalvo a qual o instrumento se destinou, como por exemplo, resistências em participar da pesquisa por fatores físicos (ex. deslocamento até o local de coleta), contribuíram para limitação da coleta. A HFS é um instrumento que pode ser respondido rapidamente quando aplicado em uma população jovem. Contudo, quando aplicada em idosos, esse tempo tornou-se mais 
longo, chegando a até uma hora de aplicação, posto que algumas questões despertavam memórias dos participantes que sentiam o desejo de compartilhá-las com o aplicador. Assim sendo, o maior tempo de aplicação, limitações relacionadas às condições físicas e sociais dos participantes e, pelo fato de sua aplicação ser individual, fez com que contribuísse para que a amostra tenha sido menor do que o esperado.

Ademais, quanto às características sociodemográficas ressalta-se a predominância do sexo feminino e do alto nível de escolaridade na amostra avaliada, evidenciando uma homogeneidade significativa. Para futuras análises de validação se mostra importante uma amostra mais heterogenia, para fins de estudo comparativo. Por fim, por se tratar de um estudo preliminar, fez-se uso de uma amostra não clínica. Considerando as especificidades características das amostras compostas por idosos, bem como o constructo em questão, sugerem-se estudos que avaliem o perdão também em amostras clínicas.

\section{Conclusão}

Com base nos resultados apresentados, verificamos que a HFS - Escala Heartland do Perdão atingiu os objetivos esperados para fins de adaptação. Esta mostrou ser um instrumento de avaliação adequado que poderá contribuir para outros estudos sobre o desenvolvimento das qualidades humanas positivas. $\mathrm{O}$ estudo do perdão em amostras de idosos permite o desenvolvimento de intervenções que possibilitem o desenvolvimento de um processo de envelhecimento mais saudável e com maior qualidade de vida. $\mathrm{O}$ investimento nestas iniciativas não só se mostra positivo para a população alvo, mas benéfico do ponto de vista de políticas públicas, potencialmente diminuindo gastos na área da saúde.

\section{Referencias}

Allemand, M. (2008). Age differences in forgivingness: The role of future time perspective. Journal of Research in Personality, 42(5), 1137-1147. doi: 10.1016/j.jrp.2008.02.009

Artes, R., \& Barroso, L. P. (2016). Introdução estatística à avaliação das escalas. In C. Gorestein, Y. Wang, \& I. Hungerbühler (Orgs.), Instrumentos de Avaliação em Saúde Mental (pp. 23-36). Porto Alegre: Artmed.

Beaton, D., Bombardier, C., Guillemin, F., \& Ferraz, M. B. (2002). Recommendations for the cross-cultural adaptation of health status measures. New York: American Academy of Orthopaedic Surgeons, 12, 1-9.

Borsa, J. C., Damásio, B. F., \& Bandeira, D. (2012). Adaptação e validação de instrumentos psicológicos entre culturas: algumas considerações. Paidéia, (53), 423432.

Cassepp-Borges, V., Balbinotti, M. A., \& Teodoro, M. L. (2010). Tradução e validação de conteúdo: uma proposta para a adaptação de instrumentos. In L. Pasquali (Org.). Instrumentação psicológica:
Fundamentos e práticas (pp. 506-520). Porto Alegre: Artmed.

Cooper, H., Camic, P., Long, D., Panter, A. T., Rindskopf, D., \& Sher, K. J. (2012). APA handbook of research methods in psychology, Vol 1: Foundations, planning, measures, and psychometrics. Washington, DC: American Psychological Association.

Cronbach, L. J. (1951). Coefficient alpha and the internal structure of tests.

Psychometrika, 16(3), 297-334.

Dahiya, R., \& Rangnekar, S. (2020).

Forgiveness in Indian organizations: a revisit of the heartland forgiveness scale. Current Psychology, 39(6), 2174-2191.

Fagundes, A. J. D. F. M. (1999). Descrição, definição e registro de comportamento. Edicon.

Gallo-Giunzioni, K., Prieto-Ursúa, M., Fernández-Belinchón, C., \& Luque-Reca, O. (2021). Measuring forgiveness: psychometric properties of the Heartland Forgiveness Scale in the Spanish population. International journal of environmental research and public health, 18(1), 45. doi: 10.3390/ijerph18010045 
Godoy Izquierdo, D., Martínez, A., \& Godoy, J. F. (2009). Balance afectivo en hombres y mujeres: Implicaciones de la edad y el sexo. Psicología Conductual, 17(2), 299319.

Ikedo, F., Castro, L., Fraguas, S., Rego, F., \& Nunes, R. (2020). Cross-cultural adaptation and validation of the European Portuguese version of the heartland forgiveness scale. Health and quality of life outcomes, 18(1), 1-9.

McCullough, M. E., \& Witvliet, C. V. (2002). The psychology of forgiveness. Handbook of positive psychology, 2, 446-455.

Organização Mundial da Saúde [OMS]. (2002). Active ageing: a police framework. Recuperado de https://apps.who.int/iris/handle/10665/6721 5

Pasquali, L. (2016). Princípios de elaboração de escalas. In C. Gorenstein, T.-P Wang, I. Hungerbühler (Orgs.), Instrumentos de avaliação em saúde mental. Porto Alegre: Artmed.

Ramírez, E., Ortega, A. R., Chamorro, A., \& Colmenero, J. M. (2014). A program of positive intervention in the elderly: Memories, gratitude and forgiveness. Aging \& mental health, 18(4), 463-470. doi: 10.1080/13607863.2013.856858

Rodrigues, G. V. A., Giacomoni, C. H., \& Argimon, I. I. L. (2019) Perdão: ressignificando vivências adversas. In I. I. L. Argimon, A. A. Moraes, C. V. Quiroga, \& G. V. A. Rodrigues (Orgs.). Avaliação e Intervenção no Ciclo Vital: da Promoção de Saúde à Prática Clínica (1 ed., pp. 181192). São Paulo: Hogrefe.

Seligman, M. E. (2002). Positive psychology, positive prevention, and positive therapy. In C. Snyder, \& S. J. Lopez (Eds.), Handbook of positive psychology (pp. 3-12). New York: Oxford University Press.

Suzman, R., Beard, J. R., Boerma, T., \& Chatterji, S. (2015). Health in an ageing world - what do we know?. The Lancet, 385(9967), 484-486.

Streiner, D. L., Norman, G. R., \& Cairney, J. (2014). Health measurement scales: a practical guide to their development and use. New York: Oxford University Press.

Thompson, L. Y., Snyder, C. R., Hoffman, L., Michael, S. T., Rasmussen, H. N., Billings, L. S., Heinze, L., Neufeld, J. E., Shorey, H. S., Roberts, J. C., \& Roberts, D. E. (2005). Dispositional forgiveness of self, others, and situations. Journal of personality, 73(2), 313-360.

Toussaint, L. L., Owen, A. D., \& Cheadle, A. (2012). Forgive to live: Forgiveness, health, and longevity. Journal of Behavioral Medicine, 35(4), 375-386. doi: 10.1007/s10865-011-9362-4

Veras, R. (2009). Envelhecimento populacional contemporâneo: demandas, desafios e inovações. Revista de saúde pública, 43, 548-554.

Hungerbühler, I. \& Wang, Y. P. (2016). Aspectos transculturais na adaptação de instrumentos. In C. Gorenstein, T.-P Wang, I. Hungerbühler (Orgs.), Instrumentos de avaliação em saúde mental. Porto Alegre: Artmed. 


\section{Dados sobre as autoras:}

- Gabriela Veiga Alano Rodrigues: Graduada em Psicologia pela Universidade da Região da Campanha (2012), possui Formação em Positive Psychology pela City University London (2014). Especialista em Religiosidade e Espiritualidade na Prática Clínica pela Pontifícia Universidade Católica do Rio Grande do Sul (2016), Mestre em Gerontologia Biomédica pela Pontifícia Universidade Católica do Rio Grande do Sul (2017) e Doutoranda em Gerontologia Biomédica pela Pontifícia Universidade Católica do Rio Grande do Sul (2019). Atualmente em Programa de Doutorado Sanduíche no Exterior (PUCRS Print) pela CAPES na Universidade Católica de Valência San Vicente Mártir (UCV) na Espanha. Pesquisadora no grupo de pesquisa AICV (Avaliação e Intervenção no Ciclo Vital) do Programa de Pós-Graduação em Psicologia da Pontifícia Universidade Católica do Rio Grande do Sul (2016).

- Carolina Villanova Quiroga: Psicóloga graduada pela Pontifícia Universidade Católica do Rio Grande do Sul (2013). Especialista em Psicologia Hospitalar (Hospital de Clinicas de Porto Alegre), com ênfase em Oncologia Pediátrica (2015). Mestre em Psicologia, com ênfase em Psicologia Clínica (PUCRS). Doutoranda em Medicina - Pediatria e Saúde da Criança (PUCRS) e membro do Developmental Cognitive Neuroscience Lab (DCNL). Especializanda em Ações Terapêuticas para Situações de Luto (PUCSP). Vice Coordenadora do Núcleo SBPH/RS (20152017). Atua em consultório particular e em Home Care. Atualmente psicóloga no Hospital de Clínicas de Porto Alegre, atuando exclusivamente na assistência em Unidade de Terapia Intensiva Covid-19.

- Irani Iracema de Lima Argimon: Irani Iracema de Lima Argimon é psicóloga (PUCRS, 1979), Especialista em Toxicologia Aplicada (PUCRS, 1990) e em Dependência Química (ABEAD, 2020). Possui Mestrado em Educação (PUCRS, 1997) e Doutorado em Psicologia (PUCRS, 2002). Terapeuta Cognitivo-Comportamental certificada pela Fundação Brasileira de Terapias Cognitivas (FBTC, 2015). Pós-doutorado em Valência - Espanha (12/2019-03/2020). É coordenadora do Grupo de Pesquisa Avaliação e Intervenção no Ciclo Vital, do PPGP da PUCRS desde 2004. Bolsista Produtividade CNPq. Professora Titular dos cursos de Graduação e de Pós-Graduação em Psicologia da PUCRS. Professora Titular do Pós-Graduação do Instituto de Geriatria e Gerontologia da PUCRS.

\section{Financiamento:}

- O presente trabalho foi realizado com apoio do Conselho Nacional de Desenvolvimento Científico e Tecnológico (CNPq) e da Coordenação de Aperfeiçoamento de Pessoal de Nível Superior - Brasil (CAPES) - Código de Financiamento 001. direitos autorais para os artigos publicados são do autor, com direitos do periódico sobre a primeira publicação. Os autores somente poderão utilizar os mesmos resultados em outras publicações indicando claramente este periódico como o meio da publicação original. Em virtude de sermos um periódico de acesso aberto, permite-se o uso gratuito dos artigos em aplicações educacionais e científicas desde que citada a fonte conforme a licença CC-BY da Creative Commons. 